\title{
Einfluss der Behandlungscompliance auf den Erfolg der Radiochemotherapie des Analkarzinoms
}

\author{
Daniel Martin ${ }^{1}$. Claus Rödel ${ }^{1}$ Emmanouil Fokas \\ Online publiziert: 19. Juni 2020 \\ (c) Der/die Autor(en) 2020
}

Hintergrund und Ziel der Arbeit Die simultane Radiochemotherapie (RCT) gilt als Standardtherapie für Patienten mit Analkarzinom, ist allerdings auch mit nicht unerheblichen Nebenwirkungen assoziiert, die u. U. eine Unterbrechung bzw. einen vorzeitigen Abbruch der RCT erfordern und dadurch die Prognose der Patienten potenziell verschlechtern. Die Autoren der hier kommentierten Arbeit untersuchten an einem großen, populationsbezogenen Patientenkollektiv die Häufigkeit von Unterbrechungen/Abbruch der RCT und deren Bedeutung für die Tumorkontrolle. AuBerdem sollten Faktoren identifiziert werden, die mit einer verminderten Compliance assoziiert sind.

Patienten und Methoden In der Ontario Cancer Registry wurden zwischen 2007 und 2015 insgesamt 1125 Patienten mit Analkarzinom identifiziert, welche eine Standard-RCT in kurativer Intention erhielten. Als Unterbrechung wurde eine Therapiepause von mehr als 7 Tagen gewertet. Die Radiotherapie galt als nichtkomplett, wenn als Gesamtdosis nicht zumindest $45 \mathrm{~Gy}$ erreicht wurden; als vollständig durchgeführte simultane Chemotherapie galten zwei Gaben von 5-Fluorouracil im Mindestabstand von 2 Wochen und zumindest eine Gabe von Mitomycin oder Cisplatin. In einer multivariablen Analyse wurden das Alter, das Geschlecht, die Komorbidität, der sozioökonomische Status, der HIV-Status sowie das Tumorstadium adjustiert.

Originalpublikation Raphael MJ, Ko G, Booth CM et al (2020) Factors Associated With Chemoradiation Therapy Interruption and Noncompletion Among Patients With Squamous Cell Anal Carcinoma. JAMA Oncol. https://doi.org/10.1001/jamaoncol. 2020.0809

$\triangle$ Dr. med. Daniel Martin

daniel.martin@kgu.de

1 Klinik für Strahlentherapie und Onkologie, Universitätsklinikum Frankfurt, Goethe-Universität Frankfurt, Theodor-Stern-Kai 7, 60590 Frankfurt, Deutschland
Ergebnisse Behandlungsunterbrechungen gab es bei 262 (23\%) der 1125 Patienten. Bei 199 (18\%) Patienten wurde nur eine Gesamtdosis von <45 Gy erreicht, und 280 (25\%) erhielten keine vollständige simultane Chemotherapie. Patienten mit höherem Komorbiditätsscore (,risk ratio“ [RR] 1,54; 95\%-CI 1,03-2,310) und solche im Alter von über 70 Jahren wiesen im Vergleich zu solchen von $<50$ Jahren (RR 0,60; 95\%-CI 0,25-0,70) eine signifikant niedrigere Wahrscheinlichkeit auf, die RCT komplett zu erhalten. Die inkomplette Durchführung der RCT, nicht aber die Unterbrechung von $>7$ Tagen war mit einem signifikant höheren Risiko für eine erforderliche abdominoperinealen Exstirpation sowie einem schlechteren krankheitsspezifischen und Gesamtüberleben assoziiert.

Schlussfolgerung der Autoren Da ein nicht unerheblicher Anteil an Patienten keine komplette RCT erhalten kann, sind Initiativen zur Verbesserung der Qualität der Behandlungsdurchführung notwendig.

\section{Kommentar}

Bei der vorliegenden Arbeit handelt es sich um eine retrospektive Auswertung eines großen Patientenkollektivs mit Analkarzinom, basierend auf dem Krebsregister von Ontario. Die Patienten rekrutierten sich aus 16 Kliniken. Auffällig ist, dass fast ein Viertel (23\%) der Patienten Behandlungsunterbrechungen von mehr als 7 Tagen aufwies und dass die Schwankungsbreite zwischen den beteiligten Kliniken diesbezüglich von 9 bis $55 \%$ reichte. Die sowieso schon relativ niedrige Gesamtdosis von $45 \mathrm{~Gy}$ als Kriterium für die „Vollständigkeit“ der RT erreichten im Gesamtkollektiv nur $82 \%$ der Patienten, erneut mit Schwankungen zwischen 66 und $93 \%$ je nach Klinik.

Dass ältere Patienten (>70 Jahre) und solche mit mehr Komorbiditäten eine niedrigere Wahrscheinlichkeit hatten, die komplette RCT zu erhalten, ist wenig überraschend. Interessanterweise bestätigt diese Arbeit aber erneut, dass eine HIV-Erkrankung nicht mit Unterbrechungen oder in- 
kompletter RCT assoziiert ist und daher die Standard-RCT bei HIV-Patienten mit Analkarzinom gemäß den aktuellen Behandlungsleitlinien ohne Einschränkung und ohne Dosiskompromisse empfohlen wird [1]. Interessant wäre gewesen, ob die Rate an Therapieunterbrechungen und -abbrüchen auch mit der jährlichen Patientenzahl pro Zentrum assoziiert ist. Ein solcher Zusammenhang wurde bereits in einer Untersuchung der amerikanischen National Cancer Database gezeigt [2], und dient als Hinweis auf den prognostischen Wert von ärztlicher Erfahrung. Weitere Gründe für die kompromittierte RCT können aufgrund der Registerdaten leider nicht genannt werden.

Aus strahlenbiologischen Erwägungen ist die Unterbrechung einer Strahlentherapie wegen des bekannten Phänomens der ,akzelerierten Repopulierung“ [3, 4] potenziell nachteilig. Dies konnte für die RCT des Analkarzinoms bei Patienten der RTOG-87-04- und RTOG-98-11-Studien gezeigt werden: Eine verlängerte Gesamtbehandlungszeit war mit einer signifikant schlechteren Prognose assoziiert [5]. Unklar ist allerdings, warum in der hier diskutierten Arbeit kein Einfluss von Therapieunterbrechungen und einer damit verbundenen Verlängerung der Behandlungszeit auf die onkologischen Endpunkte gezeigt wurde. Es ist deshalb zu mutmaßen, dass das Nichterreichen der Zieldosen zunächst der Therapieunterbrechung geschuldet war, die schließlich nicht in einem Wiederaufnehmen der Therapie mündete. Demnach kann man davon ausgehen, dass die Patienten mit Unterbrechungen trotz Pause(n) eher eine ausreichende Bestrahlungsdosis und Chemotherapiedosis erhalten hatten, was auch als signifikanter Einflussfaktor in der sekundären Auswertung der RTOG-Studie gezeigt wurde.

\section{Fazit}

Supportive Maßnahmen sind integraler Bestandteil der Behandlung von Krebspatienten [6]. Die hier kommentierte Arbeit unterstreicht dies erneut und verlangt ein engmaschiges Monitoring, damit die Notwendigkeit einer supportiven Therapie erkannt und frühzeitig eingeleitet werden kann, beispielsweise für Patienten, die wegen Analkarzinom und sicher einer Vielzahl weiterer Tumorentitäten eine RCT erhalten. Die sogenannte ,precision oncology“ mit ihrem Fokus auf eine zielgerichtete Therapie ist in Richtung einer „precision delivery“ mit Fokus auf eine adäquate Therapie zu erweitern.

\section{Daniel Martin, Claus Rödel und Emmanouil Fokas, Frankfurt/M.}

Funding Open Access funding provided by Projekt DEAL.

Interessenkonflikt D. Martin, C. Rödel und E. Fokas geben an, dass kein Interessenkonflikt besteht.

Open Access Dieser Artikel wird unter der Creative Commons Namensnennung 4.0 International Lizenz veröffentlicht, welche die Nutzung, Vervielfältigung, Bearbeitung, Verbreitung und Wiedergabe in jeglichem Medium und Format erlaubt, sofern Sie den/die ursprünglichen Autor(en) und die Quelle ordnungsgemäß nennen, einen Link zur Creative Commons Lizenz beifügen und angeben, ob Änderungen vorgenommen wurden.

Die in diesem Artikel enthaltenen Bilder und sonstiges Drittmaterial unterliegen ebenfalls der genannten Creative Commons Lizenz, sofern sich aus der Abbildungslegende nichts anderes ergibt. Sofern das betreffende Material nicht unter der genannten Creative Commons Lizenz steht und die betreffende Handlung nicht nach gesetzlichen Vorschriften erlaubt ist, ist für die oben aufgeführten Weiterverwendungen des Materials die Einwilligung des jeweiligen Rechteinhabers einzuholen.

Weitere Details zur Lizenz entnehmen Sie bitte der Lizenzinformation auf http://creativecommons.org/licenses/by/4.0/deed.de.

\section{Literatur}

1. Al-Hawary MM, Arain MA, Cooper HS (2019) NCCN Guideline Anal Cancer. https://www.ncen.org/professionals/physician_gls/ pdf/anal.pdf. Zugegriffen: 26. Sept. 2019

2. Amini A, Jones BL, Ghosh D et al (2017) Impact of facility volume on outcomes in patients with squamous cell carcinoma of the anal canal: analysis of the National Cancer Data Base. Cancer 123:228-236

3. Kim JJ, Tannock IF (2005) Repopulation of cancer cells during therapy: an important cause of treatment failure. Nat Rev Cancer 5:516-525

4. Withers HR, Taylor JM, Maciejewski B (1988) The hazard of accelerated tumor clonogen repopulation during radiotherapy. Acta Oncol 27:131-146

5. Ben-Josef E, Moughan J, Ajani JA et al (2010) Impact of overall treatment time on survival and local control in patients with anal cancer: a pooled data analysis of Radiation Therapy Oncology Group trials 87-04 and 98-11. J Clin Oncol 28:5061-5066

6. Leitlinienprogramm Onkologie (2020) Supportive Therapie. https:// www.leitlinienprogramm-onkologie.de/leitlinien/supportivetherapie/. Zugegriffen: 29. Apr. 2020 